\title{
Ytterbium-Doped Mixed Sesquioxides Grown by Pulsed Laser Deposition
}

\author{
Jake J. Prentice, James A. Grant-Jacob, Stephen J. Beecher, David P. Shepherd, Robert W. Eason, \\ Jacob I. Mackenzie \\ Optoelectronics Research Centre, University of Southampton, Southampton, SO17 1BJ, UK
}

Sesquioxides have been identified as having great potential for use as host materials for lasers due to their exceptional thermomechanical and optical properties as well as crystallographic properties well suited to the incorporation of rare-earth dopants [1]. However, fabrication of sesquioxide hosts is challenging due to their high melting points $\left(>2400^{\circ} \mathrm{C}\right)$, making it difficult to grow high-quality crystals from a melt. Pulsed laser deposition (PLD) is a well-established method for growing thin-films of a variety of materials, including doped sesquioxides, without the need for attaining such temperatures. It is also possible to exploit the non-equilibrium process to grow crystals not readily achieved via standard techniques, such as ternary or quaternary mixtures. In this paper we report the growth and characterization of $\mathrm{Yb}$-doped mixed lutetia and scandia films, which are of interest for ultrashort-pulse (USP) generation in thin-disk or waveguide lasers/amplifiers [2].

Compositional tuning of the ratio of scandia and lutetia, makes it possible to engineer the ternary crystal's lattice constant, using the <111>-plane, to lattice match to the c-cut sapphire substrate. This, coupled with a negligible difference in thermal expansion coefficients, should ensure these mixed sesquioxides grow well. Additionally, the resultant crystal being a mixture of two very different sized cations, leads to the spectroscopic properties being relatively broadband, which enables the possibility of amplifying USP sources in a compact "single-crystal" device in a thin-disk or waveguide format.

Using PLD, thin $\mathrm{Yb}_{0.1}\left(\mathrm{Lu}_{0.95-\mathrm{x}} \mathrm{Sc}_{\mathrm{x}}\right)_{2} \mathrm{O}_{3}$ films were grown on 1-mm-thick, 1-cm², sapphire substrates, heated using a $\mathrm{CO}_{2}$ laser beam to $\sim 900^{\circ} \mathrm{C}$. The ceramic targets had a composition of 5 -at $\%$ ytterbium, and $\mathrm{x}=0.45,0.55$, 0.65 . A KrF excimer laser, operating at $20 \mathrm{~Hz}$ with $20 \mathrm{~ns}$ pulses at a wavelength of $248 \mathrm{~nm}$, was used for ablating the targets in a controlled 0.01-mbar atmosphere of oxygen. The resulting thin films were analysed with darkfield microscopy, topology profiling, m-line prism-coupling, and X-ray diffraction (XRD), to determine their crystal quality. The spectroscopic properties, such as fluorescence spectrum and lifetime were also measured.
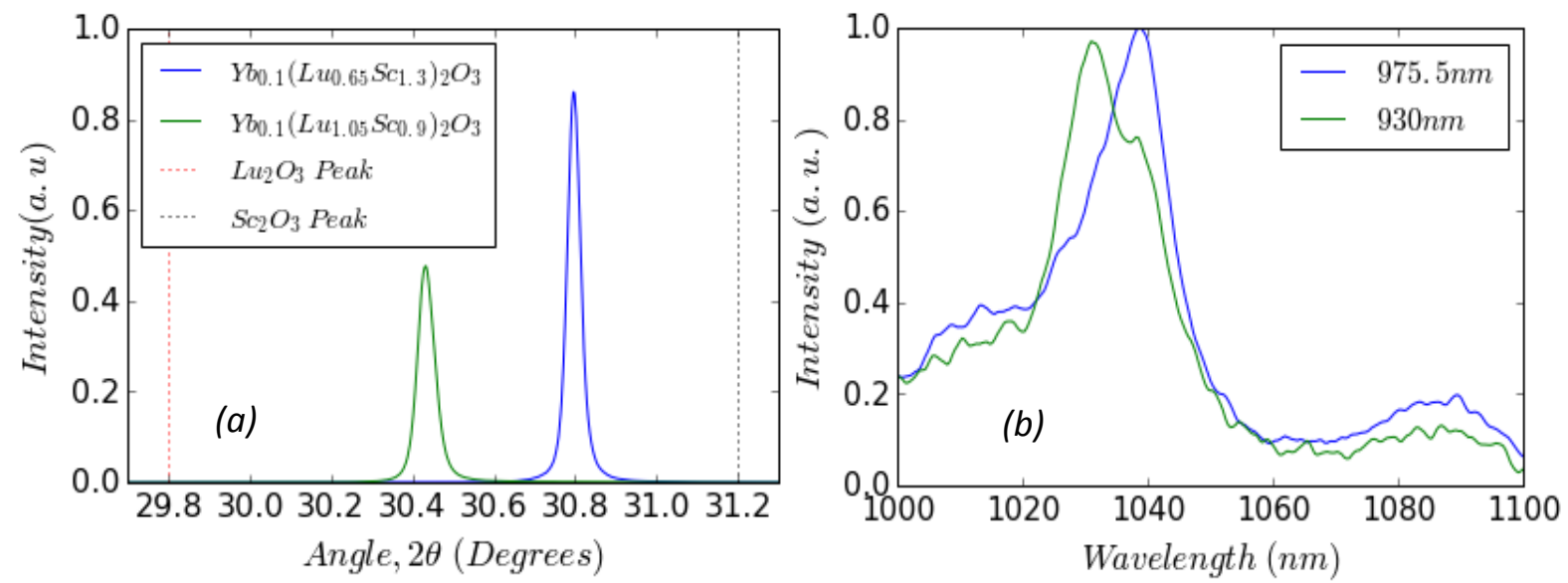

Fig 1. (a) The thin-film XRD peak for $x=0.45$ and 0.65 compositions, (b) $x=0.55$ thin-film fluorescence pumped at $976 \mathrm{~nm}$ and $930 \mathrm{~nm}$

Figure 1 (a) shows the $<222>$ mixed-sesquioxide XRD peaks for films with $\mathrm{x}=0.45$ and 0.65 , along with the theoretical peak locations for lutetia and scandia. Such narrow peaks demonstrate that the lattice constant of the sesquioxide films is defined by the compositional ratio of scandia and lutetia with a highly-ordered spacing between crystal planes. The refractive index of the film $(x=0.55)$, measured at $633 \mathrm{~nm}$ via an m-line prismcoupling system, was 1.95 , consistent with the theoretical value. Figure 1 (b) shows the fluorescence spectrum for a $1 \mu$ m-thick $\mathrm{Yb}_{0.1}\left(\mathrm{Lu}_{0.4} \mathrm{Sc}_{0.55}\right)_{2} \mathrm{O}_{3}$ film, in which the broadband emission is composed of the two main peaks associated with $\mathrm{Yb}$-doped lutetia and scandia. Fluorescence spectra from this film were observed to be dependent upon excitation wavelength, which is believed to be due to some ions occupying a lutetia-like environment while others experience a crystal field akin to a scandia-like environment. The fluorescence lifetime was measured to be $719+/-5 \mu \mathrm{s}$ (standard deviation), slightly below $850 \mu \mathrm{s}$ as reported in [1]. Further studies of these mixed sesquioxide thin films will be reported, including advances in the development of waveguide devices suitable for diode-laser-pumped lasers and amplifiers.

\section{References}

[1] C. Kränkel "Rare-Earth-Doped Sesquioxides for Diode-Pumped High-Power Lasers in the 1-, 2-, and 3- $\mu \mathrm{m}$ Spectral Range" IEEE Journal of Selected Topics in Quantum Electronics. 21, 1602013 (2015).

[2] K. Beil, C.J Saraceno, C.J Schriber, F. Emaury, O.H. Heckl, C.R. Baer,M. Golling, T. Südmeyer, U. Keller, C. Kränkel and G. Huber "Ybdoped mixed sesquioxides for ultrashort pulse generation in the thin disk laser setup." Applied Physics B, 113(1), pp.13-18. (2013) 